\title{
EDITH HEURGON
}

\section{Modéliser le fonctionnement d'une ligne d'autobus pour mieux comprendre les phénomènes d'irrégularité}

Revue française d'automatique, d'informatique et de recherche opérationnelle. Recherche opérationnelle, tome 15, no 4 (1981), p. 305-316.

<http://www.numdam.org/item?id=RO_1981_15_4_305_0>

(C) AFCET, 1981, tous droits réservés.

L'accès aux archives de la revue « Revue française d'automatique, d'informatique et de recherche opérationnelle. Recherche opérationnelle » implique l'accord avec les conditions générales d'utilisation (http://www.numdam.org/ legal.php). Toute utilisation commerciale ou impression systématique est constitutive d'une infraction pénale. Toute copie ou impression de ce fichier doit contenir la présente mention de copyright.

\section{Numdam}

Article numérisé dans le cadre du programme

Numérisation de documents anciens mathématiques

http://www.numdam.org/ 


\title{
MODELISER \\ LE FONCTIONNEMENT D'UNE LIGNE D'AUTOBUS POUR MIEUX COMPRENDRE LES PHÉNOMĖNES D'IRRÉGULARITÉ ( $\left.{ }^{*}\right)$
}

\author{
par Edith Heurgon $\left({ }^{1}\right)$ \\ sur un ensemble de travaux réalisés par: \\ Nelly Cornet, Jean-Léo Doras et Jeannine GiRard.
}

\begin{abstract}
Résumé. - Pour mieux comprendre les phénomènes d'irrégularité et tenter d'en restreindre l'ampleur, la Régie autonome des Transports parisiens a développé, depuis 1974, un vaste champ d'études combinant plusieurs modes de travail : modélisation du système dans lequel s'insère une ligne d'autobus, analyses statistiques fondées sur des enquêtes, calculs théoriques, expérimentations sur un modele de simulation.

Ces études sont succinctement décrites ici dans une double perspective: d'une part, faire ressortir l'importance et la variété des actes de modélisation qui caractérisent l'activité du chercheur opérationnel d'aujourd'hui; d'autre part, évaluer la pertinence de cette intervention en aide à la décision selon trois axes: son opérativité technique, son aptitude à être prise en charge par des acteurs, sa capacité à être mise en auvre dans l'organisation.
\end{abstract}

Mots clés : Recherche Opérationnelle, modélisation, transport, irrégularité.

Abstract. - In order to obtain a fuller understanding of the phenomena of "irregularity" and to try to limit their effects, the Paris Transport Authority has been developing since 1974 a large range of studies combining several modes of working: system modelling, statistical analyses based on surveys, theoretical calculations, and experiments using a simulation model.

These studies are concisely described here with a double aim: on one hand, to bring out the importance and variety of the modelling activities characterising the work of the operational researcher today; and, on the other, to evaluate the relevance of this decision support intervention with regard to three dimensions, operative, actorial, and organizational.

Key words: O.R., modelling, transportation, irregularity.

L'irrégularité des autobus constitue aujourd'hui, dans les villes à fort trafic, un problème essentiel pour les responsables de réseaux de transports de surface.

A partir du vaste ensemble d'études conduit sur ce thème, depuis 1974, à la Régie autonome des Transports parisiens, cet article se propose un double objectif : montrer, en premier lieu, l'importance et la variété des actes de

(*) Reçu janvier 1981 .

$\left(^{* *}\right)$ Communication présentée à la $9^{e}$ Conférence triennale sur la Recherche opérationnelle (I.F.O.R.S.) Hambourg, juillet 1981.

( $\left.{ }^{1}\right)$ Chef de la division, Recherche opérationnelle, Régie autonome des Transports parisiens.

R.A.I.R.O. Recherche opérationnelle/Operations Research, 0399-0559/1981/305/\$5.00

(C) AFCET-Bordas-Dunod 
modélisation que le chercheur opérationnel doit accomplir s'il souhaite contribuer à une meilleure compréhension des phénomènes observés; évaluer, en second lieu, la pertinence d'une telle intervention en aide à la décision selon une triple perspective : son opérativité technique, son aptitude à être prise en charge par les acteurs, sa capacité à être mise en œuvre dans l'organisation [9].

\section{LE CHAMP DES ÉTUDES SUR L'IRRÉGULARITÉ}

\subsection{La notion d'irrégularité}

A parler strict, l'irrégularité concerne les variations des intervalles réels entre les diverses voitures d'une ligne d'autobus. Mais les effets en sont perçus de manière différente selon qu'il s'agit du voyageur ou de l'exploitant : le voyageur s'attache au temps d'attente et à l'incertitude sur cette attente; l'exploitant considère en outre la variation des temps de parcours pour assurer la ponctualité des véhicules.

\subsection{Les objectifs de ces études}

Depuis 1974, la R.A.T.P. a entrepris des recherches importantes sur les phénomènes d'irrégularité. Ces études ont un double objectif :

- premièrement, un objectif de connaissance et d'interprétation des phénomènes : quels sont les facteurs qui engendrent l'irrégularité ? Comment se propage-t-elle le long d'une ligne? Quels en sont les effets pour le voyageur et pour l'exploitant? Quels sont les rapports, par exemple, entre l'irrégularité et la congestion urbaine? Et, finalement, quel est le coût de l'irrégularité pour la collectivité ?

Pour répondre, fût-ce modestement, à certaines de ces questions, il est nécessaire de mieux cerner la notion d'irrégularité et de construire des concepts permettant de la formaliser et d'en mesurer les conséquences. Car si les exploitants s'efforcent d'améliorer quotidiennement la régularité, s'ils peuvent en citer de nombreux exemples, il faut bien admettre que nulle connaissance synthétique de ce phénomène, sous une forme élaborée et surtout transmissible, n'existait au départ :

- deuxièmement, un objectif de comparaison d'actions aptes à restreindre l'irrégularité, qu'elles concernent soit l'utilisation de la voirie (couloirs, aménagement de carrefours, dispositifs de priorité aux feux, etc.), soit les méthodes et systèmes d'exploitation (construction des horaires, politiques de régulation, commandes centralisées de départ, etc.).

R.A.I.R.O. Recherche opérationnelle/Operations Research 


\section{LA PERSPECTIVE OPÉRATOIRE : MÉTHODOLOGIE GÉNÉRALE}

\subsection{Les modes de travail}

Pour aborder ce vaste champ de problèmes, plusieurs modes de travail ont été combinés : la modélisation du système, les études statistiques fondées sur des enquêtes, les calculs analytiques déduits d'hypothèses de fonctionnement, enfin l'expérimentation sur un modèle de simulation.

Ainsi, rétrospectivement, le processus d'étude (1974-1980) peut être décomposé en quatre étapes principales :

- la modélisation du système que constitue une ligne d'autobus dans un environnement urbain (1974-1975);

- les analyses statistiques et les études théoriques sur les causes et les effets de l'irrégularité (1974-1976);

- la conception et la validation du modèle de simulation PENELOPE (19761978);

- la conduite d'expérimentations et l'apprentissage de l'utilisation du modèle (1978-1980).

D'autres expérimentations sont aujourd'hui en cours de réalisation et se poursuivront, nous l'espérons, dans les prochaines années.

Revenons brièvement sur chacune de ces étapes.

\subsection{La modélisation du système}

Pour structurer le champ des études et atteindre l'objectif de connaissance, il importait, dans un premier temps, de modéliser le système que constitue une ligne d'autobus exploitée dañs un environnement urbain.

Modéliser ce système, c'est, d'une part, distinguer ses principaux composants (niveau organique); d'autre part, définir ses fonctionnements (niveau fonctionnel); enfin, retracer sa genèse (niveau génétique) $\left({ }^{2}\right)$.

Précisons ces trois niveaux dans le cas d'une ligne d'autobus.

Ses principaux composants sont des infrastructures particulières (points d'arrêt, terminus, bandes de roulement, etc.), des véhicules, du personnel (de conduite et de commande), des voyageurs, etc.

Afin de transporter des passagers d'un point à un autre d'un parcours prédéfini, ses fonctionnements résultent de l'imbrication de deux processus

$\left({ }^{2}\right)$ On retrouve ici la défınition selon trois niveaux que J.-L. Le Moigne se plaît à nommer « systémographie », dans La Théorie du système général, P.U.F., 1977, p. 38-39.

vol, $15, \mathrm{n}^{\circ} 4$, novembre 1981 
principaux (d'une part, le processus d'immobilisation aux arrêts lié au mouvement des voyageurs; d'autre part, le processus de parcours interarrêt, lié notamment aux conditions de circulation), régis par divers mécanismes de commande et de régulation (horaires, radars $\left({ }^{3}\right)$, systèmes de régulation, etc.) et par le comportement des différents protagonistes (conducteurs, contrôleurs).

Enfin, une ligne d'autobus peut aussi être caractérisée par sa genèse. Depuis sa création, et au long des années, elle accumule des expériences, discerne des spécificités, instaure des habitudes. Son exploitation - et, notamment l'établissement deses horaires - se définit non pas, à chaque fois, sur le mode d'une élaboration nouvelle, mais par de légères déformations successives qui intègrent les écarts détectés dans le fonctionnement propre du système ou dans l'environnement. C'est dire que le passé a dans son évolution un poids considérable.

Mais cette modélisation a été faite avec un projet précis : mieux comprendre les phénomènes d'irrégularité. C'est dire que, pour l'essentiel, l'attention a été focalisée sur un ensemble particulier de problèmes : les facteurs qui engendrent des variations de temps de parcours, la propagation de ces écârits le lúng de la ligne, leurs effets sur les voyageurs et le mouvement des voitures.

Nul doute, par ailleurs, que pour étudier l'évolution de l'irrégularité sur une ligne d'autobus, il faille être capable, non seulement de la représenter, mais encore de la mesurer.

La représentation la plus complète consiste, certes, en une suite d'histogrammes d'intervalles dont la déformation peut être suivie en divers points d'arrêts. Elle permet d'observer l'évolution d'un état d'équilibre instable où les intervalles sont voisins, vers un état d'équilibre stable, proche d'une arrivée au hasard des autobus.

Pour caractériser plus succinctement l'évolution de l'irrégularité le long d'une ligne d'autobus, une étude théorique (1975) a permis le choix de l'indicateur suivant :

$$
\mathrm{C}_{i}=\left(\frac{\sigma_{i}}{m_{i}}\right)^{2},
$$

$m_{i}$ et $\sigma_{i}$ désignant respectivement la moyenne et l'écart-type des intervalles à l'arrêt $i$.

On peut démontrer que cet indicateur se prête à une interprétation simple : il mesure l'accroissement relatif du temps d'attente moyen dû à l'irrégularité, c'està-dire l'attente au-delà du demi-intervalle.

( $\left.{ }^{3}\right)$ Horaire réduit, donnant au conducteur de chaque voiture, l'heure de passage aux points de régulation. 
D'autres critères peuvent, en outre, lui être associés : le pourcentage de lacunes et d'autobus en retard, le pourcentage de voyageurs refusés aux arrêts, etc.

\subsection{Les analyses statistiques et les études théoriques}

Les analyses statistiques, fondées sur des enquêtes, ont porté, pour une part, sur les facteurs d'irrégularité et, pour une autre part, sur les effets de l'irrégularité. Elles ont permis de réaliser une modélisation plus fine des principaux processus aléatoires repérés à la première étape.

Premièrement, le processus du parcours interarrêt des autobus a été décomposé en deux sous-modèles additifs :

- d'abord, le temps de parcours en régime fluide, qui tient compte des caractéristiques de la voirie : couloirs de circulation, feux, rues étroites, etc. Il est modélisé sous forme implicite par des lois gamma;

- puis, le temps de congestion, qui inclut les stationnements hors points d'arrêts, qu'ils soient dûs au niveau de la circulation ou aux perturbations comme les livraisons, etc. Il est modélisé par des lois binomiales pour l'apparition de la congestion, par des lois exponentielles pour sa valeur.

Nul doute que cette modélisation du processus de temps de parcours ne constitue une réelle originalité de l'étude.

Deuxièmement, le processus d'immobilisation aux arrêts tient compte du nombre de voyageurs montant, descendant, de la charge de l'autobus et de la proportion d'habitués de la ligne. Les arrivées de voyageurs, supposées se faire au hasard, sont modélisées par des lois de Poisson, tandis que leur destination est caractérisée par des lois multinomiales.

Les autres analyses statistiques ont porté sur les variations de temps de parcours ainsi que sur l'attente des voyageurs (réelle ou perçue). Les études théoriques ont concerné pour leur part la régulation en ligne et la dynamique des groupages d'autobus. Par ailleurs, un certain nombre d'outils statistiques ont été mis au point : générateurs de nombres pseudo-aléatoires, programmes d'analyse de la variance ou de détermination de densité de variables aléatoires, etc.

Cette deuxième étape de l'étude a contribué, en elle-même, à la satisfaction de l'objectif de connaissance. Certains résultats, permettant de formaliser et de quantifier le savoir implicite des exploitants, ont été obtenus dès ce stade [2, 5]. Donnons quelques exemples. Le modèle d'échanges de voyageurs aux arrêts a précisé l'influence du mode de compostage des billets et de l'aménagement de la voiture sur les temps de stationnement des autobus. De même, on a pu trouver les résultats suivants dont les enseignements sont d'importance : pour une ligne 
parisienne, il n'existe plus de régularité d'intervalle au-delà de $10 \mathrm{~km}$ du terminus : de plus, si l'intervalle de départ est inférieur à 3 minutes, il suffit de quelques interarrêts pour voir se former des groupages d'autobus.

Cependant, pour permettre la synthèse des différents phénomènes étudiés, pour mieux comprendre l'imbrication des causes et des effets de l'irrégularité, pour évaluer diverses actions, directes ou indirectes, susceptibles de la restreindre, a été jugée nécessaire la construction d'un modèle de simulation, fondé sur les analyses précédentes.

\subsection{La conception et la validation du modèle PENELOPE}

Ce modèle est spécialement conçu pour étudier la propagation de l'irrégularité sur une ligne d'autobus et en mesurer les conséquences.

Il simule le fonctionnement d'une ligne complète pendant toute la journée. A cette fin, il intègre la modélisation statistique des processus majeurs décrits au parâagraphe précédent, tandis que ia commande des autobus est effectuée par enchainement des départs au terminus à partir d'un horaire préétabli. Les données qui ont servi de référence à la mise au point et à la validation du modèle sont issues d'enquêtes réalisées sur la ligne 52 (Pont de Saint-Cloud - Opéra), qui comporte 74 interarrêts, 103 feux et environ $24 \mathrm{~km}$ dont $3 \mathrm{~km}$ de couloirs.

Un grand nombre de critères, concernant soit les voyageurs (temps d'attente, voyageurs refusés ou chargés, etc.), soit l'exploitation de la ligne (coefficient d'irrégularité, temps de parcours, etc.) sont calculés selon divers niveaux de détail, allant du descriptif au global.

Le modèle PENELOPE a fait l'objet d'un rigoureux étalonnage qui a permis, d'une part, d'éprouver les choix de l'assemblage et de la modélisation, d'autre part, d'évaluer la précision des résultats. La modélisation a été jugée bonne pour les études de temps de parcours (précision à $4 \%$ ) et satisfaisante pour les études d'irrégularité (plus de $80 \%$ des distributions sont convenablement reproduites, y compris les valeurs extrêmes).

Le modèle PENELOPE a fait aussi l'objet d'une validation : à partir d'autres séries de données, les ordres de grandeur des résultats du modèle et de la réalité ont été comparés. Les éléments retenus pour cette comparaison ont été la variabilité des temps de parcours totaux et l'irrégularité des intervalles à l'arrivée. Sauf certaines faiblesses (le modèle n'atteint pas les petites valeurs des temps observés), les résultats ont été jugés suffisants pour les expérimentations envisagées avec le modèle PENELOPE. 


\subsection{La conduite d'expérimentations et l'apprentissage du modèle}

Le modèle PENELOPE connaît deux principaux domaines d'intervention :

- aider à la compréhension d'un phénomène encore mal connu et qu'il a pour fonction d'explorer;

- aider à la sélection d'actions, c'est-à-dire permettre la comparaison de diverses solutions susceptibles de concourir à la réalisation de certains objectifs.

Les deux expérimentations conduites jusqu'ici avec PENELOPE ont porté, la première, sur le test de politiques de régulation (elle s'inscrit dans le domaine d'aide à la sélection d'actions) et, la seconde, sur l'étude des effets de la congestion urbaine sur l'irrégularité des autobus (elle relève du domaine d'aide à la compréhension).

Chaque expérimentation constitue, en elle-même, une étude particulière pour laquelle il convient de concevoir une méthodologie appropriée. Comme les deux premières ont été réalisées dans le cadre d'un groupe de travail associant, d'une part, les chargés d'études d'exploitation de la direction du réseau routier et, d'autre part, les chercheurs opérationnels de la direction des études générales, le processus d'étude suivant a été mis au point :

(a) demande formulée par l'utilisateur, à l'aide d'un document de deux pages justifiant de l'intérêt de l'étude et en précisant les grandes lignes;

(b) configuration du champ de l'expérimentation par le groupe de travail, c'està-dire formulation des questions auxquelles l'étude tente d'apporter des réponses et examen de la précision que l'on peut escompter des résultats du modèle face à tel champ de problèmes;

(c) constitution d'un plan d'expériences, c'est-à-dire d'une série de variantes contrastées obtenues en jouant sur les paramètres d'entrée, qu'ils concernent l'environnement (voyageurs, feux, conditions de circulation, etc.) ou la commande des autobus (horaires, politiques de régulation, etc.);

(d) réalisation du plan d'expériences, en observant l'évolution du modèle dans chaque cas, " toutes choses étant égales par ailleurs »;

(e) interprétation des résultats dans le cadre d'une discussion fournie qui peut conduire soit à de nouvelles expériences, soit à la rédaction d'un rapport de synthèse qui dégage des conclusions de portée plus générale.

Selon le type d'études, l'ampleur de chaque phase comme l'interaction entre les différentes étapes peuvent varier de manière significative. Par exemple, dans le cas assez concret du test de politiques de régulation, les phases (a) et (b) ont pu être réalisées assez rapidement et, à l'issue de la phase (e), peu d'itérations ont été nécessaires. En revanche, pour l'étude sur la congestion, une longue procédure de recherche a seule permis la configuration progressive du champ de 
l'expérimentation en faisant varier les différents paramètres capables d'introduire la congestion dans le modèle, afin de repérer certaines formes de congestion concrètement identifiables (une rue bloquée par un camion, un carrefour encombré, un passage difficile).

Utilisé dans une telle perspective, le modèle manifeste une véritable fonction heuristique, c'est-à-dire qu'il permet de formuler des problèmes en les faisant apparaitre d'abord artificiellement, puis en les confrontant à la pratique des exploitants.

Donnons rapidement une idée des résultats qui ont pu être obtenus à l'issue de ces deux premières expérimentations.

La première expérimentation a confirmé que, du point de vue des voyageurs, une régulation en ligne pouvait venir compléter efficacement l'actuelle régulation de terminus. Elle a fait apparaître, par ailleurs, du point de vue des coûts d'exploitation, une certaine prédominance de la régulation d'horaire par rapport à la régulation d'intervalle, à condition de pouvoir assurer une bonne qualité de l'horaire dont l'établissement suppose une précise connaissance des

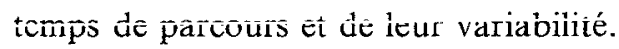

La seconde expérimentation a permis la mise en évidence de certains résultats, de nature parfois qualitative. Par exemple : la congestion n'est pas le seul facteur d'irrégularité; en outre, la présence d'un seul " point noir », quelle que soit son ampleur, introduit une irrégularité significative sur une ligne auparavant régulière. De plus, l'irrégularité acquise en un point de la ligne se conserve jusqu'à la fin du parcours, en l'absence d'intervention externe. Enfin, il s'avère qu'un point noir doit être traité jusqu'à sa disparition totale et qu'il ne peut être combattu indépendamment des perturbations voisines, une ligne d'autobus constituant un système complexe dont il convient d'appréhender simultanément les divers composants.

Mais cette expérimentation a fourni parallèlement certaines indications quantitatives: par exemple, si une perturbation de 10 minutes affecte un autobus sur dix, l'augmentation de temps de parcours moyen qui en résulte par course est due pour un tiers à la valeur de la congestion, pour un autre tiers au temps de stationnement supplémentaire aux arrêts et pour le dernier tiers au ralentissement induit par le groupage des autobus.

La conduite de ces deux premières expérimentations a permis un certain apprentissage de l'utilisation du modèle par le groupe de travail, tant au niveau de la configuration des problèmes, qu'à celui de la définition des plans d'expérience et de l'interprétation des résultats. L'étude de sensibilité que constitue la seconde expérimentation a montré, par exemple, que l'extrême précision de la modélisation de la congestion à l'entrée n'était peut-être pas nécessaire en raison

R.A.I.R.O. Recherche opérationnelle/Operations Research 
de la faible variabilité des résultats globaux de la simulation. La compréhension de tels phénomènes a conduit à faire des hypothèses plus fortes pour la conception d'un autre modèle de simulation, visant cette fois à étudier la propagation de l'irrégularité sur une ligne de métro.

Pour clore cette partie méthodologique et en référence au titre même de cet article, il semble opportun de faire la remarque suivante : divers actes de modélisation ont caractérisé ces études. D'une part, ce que nous avons appelé la modélisation systémique; d'autre part, à un niveau plus fin, la modélisation statistique ou analytique des divers processus ou phénomènes; enfin, la conception d'un modèle de simulation. De plus, chaque expérimentation exige une modélisation préalable du problème qu'elle envisage de traiter.

Il est clair qu'à chaque fois le terme de modélisation est à entendre dans un sens plus ou moins étendu, tantôt systémique, tantôt statistique, tantôt analytique, tantôt informatique... Mais ce qu'il convient de faire paraître ici, c'est, selon nous, malgré ces différences, l'importance et la variété de la modélisation dans l'activité du chercheur opérationnel d'aujourd'hui.

\section{LES TROIS AXES DE PERTINENCE D'UNE INTERVENTION EN RECHERCHE OPÉRATIONNELLE}

Jusqu'ici, s'agissant de nos études sur l'irrégularité des autobus, nous avons examiné principalement l'axe de la pertinence opératoire [9], c'est-à-dire l'aptitude des études à contribuer à la formulation et à la résolution du problème considéré.

Mais, pour évaluer une intervention de la recherche opérationnelle dans une organisation, il importe de considérer aussi sa pertinence actoriale et sa pertinence organisationnelle.

Par pertinence actoriale, nous entendons l'aptitude d'une étude à être prise en charge par un acteur (individuel ou collectif), ou par un système d'acteurs de l'organisation, qui en « fait son affaire ». Les conditions de possibilité d'une telle prise en charge nous paraissent être les suivantes : premièrement, l'acteur doit disposer de compétences à l'égard du problème traité de manière à être en mesure de s'approprier l'étude; deuxièmement, il doit éprouver de réelles motivations visà-vis du projet, compte tenu éventuellement de sa stratégie personnelle, de façon à pouvoir s'y impliquer largement; troisièmement, il doit être doté de movens et de poucoirs suffisants dans l'organisation pour mener à terme sa mission.

Par pertinence organisationnelle, nous entendons l'aptitude d'une étude, conçue comme un processus de changement, à s'insérer effectivement dans vol. $15, \mathrm{n}^{\circ} 4$, novembre 1981 
l'organisation, sans y commettre d'irréversibles effets pervers et de façon à y accroître l'intelligence collective.

Dans le cadre de cet article, nous ne sommes pas en mesure d'examiner complètement et de manière argumentée tous les problèmes relatifs à ces deux types de pertinence. Néanmoins, quelques remarques, sans doute encore provisoires, sont aptes à en donner quelque idée.

\subsection{La pertinence actoriale}

S'agissant de pertinence actoriale, il convient, d'abord, de repérer dans l'organisation les divers acteurs susceptibles de prendre en charge un stade ou un autre du projet, c'est-à-dire qui réunissent, éventuellement à des degrés divers, des compétences (théoriques ou pratiques), des motivations, des moyens et du pouvoir.

Outre l'équipe de recherche opérationnelle qui a conçu et réalisé une large tranche des travaux mentionnés ci-dessus, plusieurs autres acteurs sont concernés directement par ces études sur l'irrégularité des autobus.

Il y a, en premier lieu, le service de l'exploitation du réseau d'autobus. En effet, ce sont les opérationnels chargés de l'exploitation quotidienne des lignes qui ont la plus grande expérience des phénomènes observés. Nul doute, d'une part, que de la confrontation des résultats des études et de leur connaissance du terrain, ils puissent tirer de fructueux enseignements : tantôt la confirmation plus formalisée de leur savoir implicite, tantôt la clarification de certaines notions contre-intuitives (dans le domaine des phénomènes aléatoires, par exemple), tantôt, même, des règles d'action infléchissant leurs conduites coutumières. Nul doute, d'autre part, que la confrontation de l'expérience des exploitants et des résultats des modèles, ne permette aux hommes d'étude de faire progresser leurs recherches.

Mais ces études intéressent, en second lieu, le service des études du réseau d'autobus qui a pour charge, notamment, d'euvrer au perfectionnement des méthodes et des systèmes d'exploitation et de négocier avec les autorités publiques certains aménagements de la voirie ou de la réglementation aptes à améliorer la circulation des autobus.

Les travaux sur l'irrégularité ont été conduits dans le cadre d'une collaboration, plus ou moins étroite, entre chargés d'études d'exploitation et chercheurs opérationnels. Si la prise en charge des premières phases a été assurée principalement par les chercheurs opérationnels, il semble bien qu'aujourd'hui, dépassant le stade du simple soutien intellectuel, le service des études du réseau routier soit en train de prendre progressivement la relève. Il convient d'admettre cependant que, jusqu'ici, l'implication des opérationnels a été trop restreinte et 
qu'un travail d'envergure reste à accomplir à cet égard, avec la médiation nécessaire des chargés d'études d'exploitation.

\subsection{La pertinence organisationnelle}

Parfois, malgré une réelle pertinence actoriale, certaines études se heurtent, au moment de leur mise en œuvre dans l'organisation, à des obstacles considérables. Parfois, aussi, elles produisent des effets, directs ou indirects, bien différents de ceux qui étaient escomptés.

N'en doutons pas : toute étude constitue, à un degré plus ou moins fort, un possible facteur de changement. Par là même, elle déclenche dans l'organisation de virulentes forces adverses. Des structures rigides, des fonctionnements automatiques, des privilèges menacés, peuvent être à l'origine de résistances que seule une vigilante stratégie de développement est apte à vaincre.

$1 l$ est sans doute trop tôt pour pouvoir évaluer, selon la perspective organisationnelle, nos études sur l'irrégularité : leur insertion, en effet, n'est pas encore accomplie.

On peut avouer cependant que jusqu'ici leur stratégie de développement n'a pas été très maîtrisée. Elle s'est souvent élaborée au coup par coup et la méthodologie générale présentée dans la première partie de cet article constitue une sorte de rationalisation a posteriori d'un processus d'étude plutôt chaotique. Malgré cette insuffisance méthodologique, les chances de succès de la mise en œuvre ne semblent pas encore compromises.

Outre son aptitude à s'insérer dans l'organisation, la pertinence organisationnelle d'une étude se mesure aussi par sa faculté à accroître ce qu'on peut appeler l'intelligence collective de l'organisation. Il nous paraît qu'à leur façon les études sur l'irrégularité disposent, à cet égard, d'une pertinence certaine. Par le long processus d'apprentissage qui les caractérise, elles peuvent contribuer à instaurer, de part et d'autre de l'entreprise, des références communes, des savoirs partagés, des langages plus proches, des méthodes de travail plus efficaces. Nul doute que le potentiel intellectuel de l'organisation ne s'en trouve ainsi significativement accru.

\section{CONCLUSION}

L'évaluation des études d'irrégularité selon les trois axes de pertinence peut être résumée ainsi : leur pertinence opératoire est bonne (elles sont aptes à contribuer à une meilleure connaissance des phénomènes et facilitent le choix d'actions); leur pertinence actoriale, en revanche, a besoin d'être largement 
accrue, surtout du côté des opérationnels; enfin, du point de vue de la pertinence organisationnelle, le travail reste encore, pour une grande part, à accomplir.

Seule la conjonction d'un bon niveau de pertinence sur les trois axes permettra, nous semble-t-il, d'obtenir pour l'entreprise de transport, la fructification maximale des potentialités offertes par ce vaste champ d'études.

\section{BIBLIOGRAPHIE}

1. N. Cornet, J.-L. Doras et J. Grrard, Modèle de simulation d'une ligne d'autobus (PENELOPE), Rapport interne R.A.T.P. GE 787146, avril 1978.

2. N. Cornet, J.-L. Doras, J. Girard et R. Besso, Tests de politique de régulution ate'c le modèle PENELOPE, Rapport interne R.A.T.P. GE 807057, février 1980.

3. J.-L. DoRAS, Montée et descente des voyageurs aux arrêts d'autobus, Revue TEC, vol. 10, 1975.

4. J.-L. Doras, M. Girardot et E. Heurgon, Pratique de la simulation à la R.A.T.P. résultats, enseignements, perspectives, Revue Automatisme, mai 1978.

5. J.-L. Doras, Irrégularité des autobus et temps d'attente des voyageurs, Revue T.E.C., vol 33, 1979, p. 13-21.

6. J.-L. Doras, J. GiRARD et R. Besso, Effets de la congestion sur une ligne d'autobus (test arec le modèle PENELOPE), Rapport interne R.A.T.P. GE 807239, juillet 1980.

7. E. HeURGon, Penser la recherche opérationnelle autrement, Informatique et gestion, vol. 105, avril 1979, p. 48-51.

8. E. Heurgon, OR in the firm: an alternative design, E.J.O.R., vol. 7, 1981, p. 183-190.

9. E. HeURGON, Les rapports du processus d'étude et du processus de décision dans l'aide à la décision, Communication au congrès EURO, juillet 1980 (à paraitre). 